\title{
Not easy to become a tumor marker
}

\author{
Bogdan Solnica \\ Department of Clinical Biochemistry, Jagiellonian University Medical College, Kraków, Poland
}

Correspondence to: Bogdan Solnica, MD, PhD, Department of Clinical Biochemistry, Jagiellonian University Medical College, ul. Kopernika 15A 31-501 Kraków, Poland phone: +481242140 06 e-mail: mbsolnic@cyf-kr.edu.pl Received: November 13, 2016 Accepted: November 14, 2016 Published online: November 30, 2016 Conflict of interests: none declared. Pol Arch Med Wewn. 2016. 126 (11): $845-846$ doi:10.20452/pamw.3699 Copyright by Medycyna Praktyczna Kraków 2016
A tumor marker is a substance produced by or associated with a tumor, found in blood in concentrations higher than in malignancy-free subjects. ${ }^{1}$ The long history of tumor markers is a little disappointing. They were introduced on a larger scale in the years 1960-1970 with the hope that they would enable the diagnosis of malignancies at an early stage and serve as screening tests. In fact tumor markers are mainly used to monitor the course of the cancer disease after radical treatment. Rising concentrations of a tumor marker is an earlier indicator of cancer recurrence or distant metastases than clinical presentation and results of imaging studies are. Only a few tumor markers are used for screening (eg, alpha-fetoprotein in patients at risk for hepatocellular carcinoma or prostate-specific antigen together with other diagnostic tools to screen for prostate cancer) or for disease staging and prognosis. ${ }^{1}$ Currently, molecular genetic tumor markers including cell-free DNA seem to open up new perspectives. ${ }^{2,3}$ Due to still unsatisfactory detection rates of cancer at an early stage and limitations of currently available tumor markers, numerous candidate markers are evaluated in terms of specificity for tumor and diagnostic characteristics.

A good example of such an evaluation is a paper by Łukasiewicz-Zając et $\mathrm{al}^{4}$ reporting serum concentrations of receptor for interleukin 8 (IL-8) in patients with esophageal cancer (EC), published in the current issue of the Polish Archives of Internal Medicine (Pol Arch Med Wewn). In cancer, the immune response of the body is altered resulting in inflammation, angiogenesis, and suppression of cancer immunosurveillance. ${ }^{5}$ Cytokines, chemokines, and their receptors are involved in this process. ${ }^{5,6}$ CXCR2, an IL-8 receptor, is expressed in several human carcinomas including EC, and the IL-8/CXCR2 signaling pathway enhances cancer development by promotion of proliferation, invasion, and metastases of cancer cells, and angiogenesis within the tumor. ${ }^{6}$

High expression of CXCR2 on EC cells has been reported to be associated with malignancy progression and poor prognosis. ${ }^{78}$ Łukasiewicz-Zając et $\mathrm{al}^{4}$ looked at serum CXCR2 concentrations in
EC patients as an alternative to the labor-intensive and expensive immunohistochemical procedures used to detect this chemokine receptor expression on cancer cells. Thus, the authors assumed a relationship between the degree of local CXCR2 expression on EC cells and its serum concentration. Indeed, they found significantly higher serum CXCR2 levels in EC patients than in healthy controls. However, their study failed to show any relationship between serum CXCR2 concentrations and the clinical and pathological EC characteristics, found in studies on the expression of CXCR2 on EC cells. ${ }^{7.8}$ The authors emphasized that they carried out the first study evaluating serum CXCR2 concentrations in EC patients. Therefore, they could not present any data on the relationship between serum CXCR2 and its expression on EC cells. There are also no such data for other cells expressing CXCR2. Soluble CXCR2 (sCXCR2), present and measurable in biological fluids (plasma, urine), is an acidic glycopeptide identified as the $\mathrm{N}$-terminal fragment of the CXCR2 molecule. It is released after proteolytic cleavage of CXCR2 by cell-associated metalloproteinases as shown for neutrophils. ${ }^{9}$ Thus, the relationship between serum sCXCR2 and CXCR2 expression may be complex.

The second aim of the study was to establish the diagnostic characteristics of CXCR2 levels in the diagnosis of EC and to assess the clinical usefulness of this test. The obtained results clearly demonstrated that significant differences in serum CXCR2 levels between EC patients and tumor-free controls do not justify the measurement of this receptor to diagnose EC. CXCR2 is expressed on numerous cells, namely, on neutrophils, lymphocytes, as well as epithelial and endothelial cells, and cannot be considered a tumor-specific marker. Therefore, its serum concentration distributions in EC patients and healthy controls may overlap. The area under the receiver-operating characteristic curve (AUC) reflects the degree of overlapping of distributions. AUC for serum CXCR2 levels amounting to 0.681 found in the study indicates significant overlapping of distributions and poor overall diagnostic 
performance of this test. Similarly, other parameters of diagnostic characteristics (sensitivity, specificity, predictive values of results) found in the study do not support the use of serum CXCR2 in the diagnosis of EC.

Assessment of diagnostic accuracy is considered the first step in evaluating a candidate marker. Diagnostic accuracy should be linked to clinical utility defined as the degree to which the actual use of the evaluated test is associated with changing health outcomes. ${ }^{10}$ The assessment of clinical utility should be based on the added value of the test and the results of validation and cost-effectiveness studies. ${ }^{11}$ These steps of evaluating candidate markers seem to be sometimes overlooked.

Thus, even the cautious conclusion of the authors about the potential use of serum CXCR2 levels in the diagnosis of EC seems to be too optimistic, but, indeed, further studies addressing this issue are required.

\section{REFERENCES}

1 Chan DW, Booth RA, Diamandis EP. Tumor Markers. In: Burtis CA, Ashwood ER, Bruns DE, eds. Tietz Fundamentals of Clinical Chemistry. St. Louis, Missouri: Saunders Elsevier; 2008: 337-362.

2 Tie J, Gibbs P. Sequencing Circulating Cell-Free DNA: the potential to refine precision cancer medicine. Clin Chem. 2016; 62: 796-798.

3 Leung F, Kulasingam V, Diamandis EP, et al. Circulating tumor DNA as a cancer biomarker: Fact or Fiction? Clin Chem. 2016; 62: 1054-1060.

4 Łukaszewicz-Zając M, Kulczyńska-Przybik A, Muszyński P, et al. Serum concentrations of receptor for interleukin 8 in patients with esophageal cancer. Pol Arch Med Wewn. 2016; 126: 854-861.

5 Burkholder B, Huang RY, Burgess R, et al. Tumor-induced perturbations of cytokines and immune cell networks. Biochim Biophys Acta. 2014; 1845: 182-201.

6 Verbeke H, Geboes K, Van Damme J, Struyf S. The role of CXC chemo kines in the transition of chronic inflammation to esophageal and gastric cancer. Biochim Biophys Acta. 2012; 1825: 117-129.

7 Nishi T, Takeuchi H, Matsuda S, et all. CXCR2 expression and postoperative complications affect long-term survival in patients with esophageal cancer. World J Surg Oncol. 2015; 13: 232

8 Sui , Hu P, Zhang T, et al. High expression of CXCR-2 correlates with lymph node metastasis and predicts unfavorable prognosis in resected esophageal carcinoma. Med Oncol. 2014; 31: 809.

9 Katlinski K, Akalovich S, Katlinskaya Y, et al. Soluble human CXCR2: dtructure, properties, bioactivity. Cytokine. 2009; 48: 2 .

10 Bossuyt PM, Reitsma JB, Linnet K, Moons KG. Beyond diagnostic accuracy: the clinical utility of diagnostic tests. Clin Chem. 2012; 58 1636-1643

11 Moons KG. Criteria for scientific evaluation of novel markers: a perspective. Clin Chem. 2010; 56: 537-541. 